\title{
Inflammatory agents partially explain associations between cortical thickness, surface area and body mass in adolescents and young adulthood.
}

Prats-Soteras, X. ${ }^{a, b, c}$; Jurado, M.A. ${ }^{a, b, c}$; Ottino-González, J. J,b,c ; García-García, I. ${ }^{\text {; }}$; Segura, B. ${ }^{\mathrm{b}, \mathrm{e}}$; Caldú, X. ${ }^{\mathrm{a}, \mathrm{b}, \mathrm{c}}$; Sánchez-Garre, C. ${ }^{\mathrm{f}, \mathrm{h}}$; Miró, N. ${ }^{\mathrm{f}, \mathrm{h}}$; Tor, C. ${ }^{\mathrm{g}, \mathrm{h}}$; Sender-Palacios, M.g.h ; Garolera, M. ${ }^{\text {h,i }}$

a Departament de Psicologia Clínica i Psicobiologia, Universitat de Barcelona, Spain

${ }^{\mathrm{b}}$ Institut de Neurociències, Universitat de Barcelona, Spain

`Institut de Recerca Sant Joan de Déu, Hospital Sant Joan de Déu, Spain

${ }^{\mathrm{d}}$ Montreal Neurological Institute, McGill University, Canada

e Departament de Medicina, Universitat de Barcelona, Barcelona, Spain

${ }^{f}$ Pediatric Endocrinology Unit, Hospital de Terrassa, Consorci Sanitari de Terrassa, Spain

${ }^{g}$ CAP Terrassa Nord, Consorci Sanitari de Terrassa, Barcelona, Spain

h Brain, Cognition and Behavior Clinical Research Group, Consorci Sanitari de Terrassa, Barcelona, Spain

' Unitat de Neuropsicologia, Hospital de Terrassa, Consorci Sanitari de Terrassa, Barcelona, Spain

Running title: Inflammation, gray matter and BMI in adolescence

Corresponding author:

Name: Dr. Maria Angeles Jurado

Postal address: Pg. Vall d'Hebron 171 (08035 Barcelona)

Telephone number: +34 933125055

E-mail: majurado@ub.edu

Word count: 4,397

Declarations of interest: none 


\section{ABSTRACT}

Background/Objectives: Excessive body mass index (BMI) has been linked to a low-grade chronic inflammation state. Unhealthy BMI has also been related to neuroanatomical changes in adults. Research in adolescents is relatively limited and has produced conflicting results. This study aims to address the relationship between BMI and adolescents' brain structure as well as to test the role that inflammatory adipose-related agents might have over this putative link.

Methods: We studied structural MRI and serum levels of interleukin-6, tumor necrosis factor alpha (TNF- $\alpha$ ), C-reactive protein and fibrinogen in 65 adolescents (aged 12-21 years). Relationships between BMI, cortical thickness and surface area were tested with a vertex-wise analysis. Subsequently, we used backward multiple linear regression models to explore the influence of inflammatory parameters in each brain-altered area.

Results: We found a negative association between cortical thickness and BMI in the left lateral occipital cortex (LOC) and the right precentral gyrus as well as a positive relationship between surface area and BMI in the left rostral middle frontal gyrus and the right superior frontal gyrus. In addition, we found that higher fibrinogen serum concentrations were related to thinning within the left LOC $(\beta=-0.45, p<0.001)$, while higher serum levels of TNF- $\alpha$ were associated to a greater surface area in the right superior frontal gyrus $(\beta=0.32, p=0.045)$. Besides, we have also identified a trend that negatively correlates the cortical thickness of the left fusiform gyrus with the increases in BMI. It was also associated to fibrinogen ( $\beta=-0.33, p=0.035)$.

Conclusions: These results suggest that adolescents' body mass increases are related with brain abnormalities in areas that could play a relevant role in some aspects of feeding behavior. Likewise, we have evidenced that these cortical changes were partially explained by inflammatory agents such as fibrinogen and TNF- $\alpha$. 


\section{INTRODUCTION}

Overweight and obesity have become a pandemic. Child and-youth obesity are rising at an alarming rate. According to the World Health Organization (WHO), over 340 million children and adolescents aged 5-19 were overweight or obese in 2016 [1]. This is a worldwide source of concern because excessive weight during youth has been associated with an increased incidence of cardiometabolic diseases (e.g., type II diabetes, stroke, and hypertension), some types of cancer, and premature mortality in adulthood [2].

Excessive weight has been related to neuroanatomical changes in adults. Mainly, increases in body mass index (BMI) have been related to a wide-spread pattern of cortical gray matter volume (GMV) reductions [3]. In pediatric populations, although scarce, findings are in line with those described in adults, mostly in frontal and limbic regions $[4,5]$.

Despite cortical GMV has often been used to measure gray matter density of the cortical mantle, it is worth noting that cortical GMV can be regarded as the product of thickness and surface area $[6,7]$. For this reason, analyzing cortical thickness and surface area separately could improve the specificity of the results, since a loss of GMV may reflect either reduced thickness, reduced area, or both [8]. Cortical thinning could provide some indication of neural loss, reduced size of neural cell bodies or degradation, and could be influenced by white matter properties, such as myelination [9]. On the other hand, surface area measures could mirror the tension or shrinkage of underlying white matter fibers [10]. In addition, these two constitutive components of cortical volume seem to be influenced by different genetic and cellular processes and follow distinct patterns of development [11]. Therefore, the study of cortical thickness and surface area separately can provide more accurate information about gray matter changes.

To date, studies in adolescents assessing the relationship between cortical thickness and body weight are scarce and have produced conflicting results. While two studies showed a negative link between obesity and cortical thickness $[12,13]$, others found no relationship with obesity or BMI [14-17]. Notwithstanding, one of these works that did not identify any effect of BMI on healthy adolescents' cortical thickness observed a significant age-by-BMI z-score interaction related to altered cortical thickness across development [17]. Likewise, it is also worth pointing the recent study of Ronan and colleagues [18], since they found that increased BMI was associated with reduced cortical thickness in several regions of the prefrontal cortex in a sample of 2700 healthy children aged between 9 and 11 . Regarding the association between BMI and surface area, only one study has examined it, which yielded null results [16].

Excessive weight is related to a low-grade chronic inflammation state $[19,20]$ that might potentially lead to oxidative stress scenarios [21]. Both hypertrophied adipocytes and adipose tissue-resident macrophages produce inflammatory mediators such as interleukin-6 (IL-6), tumor necrosis factor alpha (TNF- $\alpha$ ), C-reactive protein (CRP) and fibrinogen [22-25]. Although a healthy brain is an immune-privileged organ due to the blood-brain barrier shielding properties [26], adipose-related inflammatory by-products can cross and disrupt its permeability, amplifying and sustaining a chronic inflammatory milieu $[21,27,28]$.

There is evidence that points to a correlation between the levels of cytokines in blood and in the cerebrospinal fluid [29]. Elevated levels of peripheral pro-inflammatory agents could suggest a neuroinflammatory state, which in turn could be involved in potential cortical brain 
abnormalities BMI-related. In fact, one study with middle aged and elderly people reported that adiposity-related systemic inflammation may be related with brain structures [30].

As abovementioned, research exploring neuroanatomical correlates of BMI in adolescents is relatively limited, particularly in terms of cortical thickness and surface area. Our study has two aims: 1 ) to address the relationship between BMI and adolescents' brain structure and 2) to test if this relationship could be explained by adipose-related levels of inflammatory biomarkers. We expect to find cortical changes related to an increase in BMI. Likewise, we assume that such connection will be partially explained by the presence of inflammatory agents.

\section{MATERIALS AND METHODS}

\section{Participants}

This study includes 65 adolescents (age $=15.89 \pm 2.72$ years; 33 females), who were recruited from public primary care centers belonging to the Consorci Sanitari de Terrassa (Spain). All of them met two inclusion criteria: (1) being aged from 12 to 21 years old and (2) having a sex and age-specific BMI over the $5^{\text {th }}$ percentile according to the 2000 Centers for Disease Control and Prevention (CDC) growth charts [31]. Volunteers older than 17 had to show a BMI higher than $18.5 \mathrm{~kg} / \mathrm{m}^{2}$.

Individuals who met inclusion criteria and agreed to participate underwent a medical evaluation and a blood-sample, both performed in the Pediatric Endocrinology Unit at the Hospital de Terrassa in adolescents younger than 19 years old, and in the CAP Terrassa Nord for older participants. In this visit, participants' clinical history was reviewed and anthropometric measures (i.e., weight and height) were taken to exclude those who presented (1) history of psychiatric illness (including eating disorders such as bulimia or binge eating disorder), (2) history of regular drug use (3) developmental, (4) neurological, or (5) systemic disorders (e.g., hyper or hypothyroidism and diabetes). Additionally, (6) meeting metabolic syndrome criteria [32] also was an exclusion criterion for participants older than 17 years old. The presence of metabolic syndrome in adolescents under 18 years of age was not taken into account because there is no general consensus to define pediatric metabolic syndrome [33].

Participants who did not have any medical exclusion criteria went through a neuropsychological assessment to discard (7) the presence of global cognitive impairment (scalar score lower than 7 in vocabulary subtests of Wechsler batteries $[34,35]$ was interpreted as an estimated IQ below 85). Finally, adolescents who did not present any of the exclusion criteria abovementioned were proposed to undergo a magnetic resonance imaging (MRI). For this work, only those who accepted undertake this procedure were included in the study.

The final sample was composed by 65 adolescents. The flow of included and excluded participants is detailed in depth in Supplementary Material section (Appendix A.1). An overview of demographic, anthropometric and inflammatory characteristics is shown in Table 1.

This study was approved by the Institutional Ethics Committee of the University of Barcelona (CBUB); Institutional Review Board (IRB 00003099, assurance number: FWA00004225; http:// 
www.ub.edu/recerca/comissiobioetica.htm). The research was conducted in accordance with the Helsinki Declaration. Written informed consent was obtained from each participant (if older than 18 years old) or their parents prior to taking part in the study and after explaining the research purpose and procedures.

\section{Image acquisition}

Sixty-five adolescents underwent an MRI acquisition on a 3T MAGNETON Trio (Siemens, Germany) at the Institut d'Investigacions Biomèdiques August Pi I Sunyer (IDIBAPS) from the Centre de Diagnòstic per la Image Clínic (CDIC). We acquired a high resolution T1-weighted 3D using a Magnetization Prepared Rapid Acquisition Gradient echo (MPRAGE) sequence with the following parameters: repetition time $2300 \mathrm{~ms}$; echo time $2.98 \mathrm{~ms}$; inversion time $900 \mathrm{~ms}$. A total of 240 contiguous $1-\mathrm{mm}$ slices were acquired using a $256 \times 256$ matrix with an in-plane resolution of $1 \times 1 \mathrm{~mm}^{2}$. The MRI acquisitions took place around 3 weeks later of blood extractions.

\section{Data processing}

We performed the preprocessing and analysis of cortical thickness and surface area using standard procedures in FreeSurfer software (Version 6.0) (https://surfer.nmr.mgh.harvard.edu). Briefly, this process included motion correction and T1 averaging [36], removal of non-brain tissue [37], intensity normalization [38], cortical parcellation [39] and tessellation of gray/white matter tissue. Cortical thickness is represented by the distance between gray/white boundary and the gray/cerebrospinal fluid boundary at each vertex on the surface [40]. After Freesurfer preprocessing, results for each subject were visually inspected to ensure accuracy of registration, skull stripping, segmentation, and cortical surface reconstruction. Small imprecisions were detected across all participants. Consequently, errors were fixed by manual intervention following standard procedures (https://surfer.nmr.mgh.harvard.edu/fswiki/FsTutorial/TroubleshootingData\#Fixingerrors).

\section{Inflammation biomarkers}

Blood samples were obtained from each participant after overnight fasting state to obtain serum levels of pro-inflammatory biomarkers (TNF- $\alpha$, IL-6, high-sensitivity C-reactive protein (hs-CRP) and fibrinogen). This procedure was performed between 8:00 and 8:30 AM in the Pediatric Endocrinology Unit at the Hospital de Terrassa in participants younger than 19 and in the CAP Terrassa Nord for older volunteers. Blood samples were preserved at $-80^{\circ} \mathrm{C}$ at the Logistics Park of Health (CatLab) until analyzed. Serum concentrations of hs-CRP and fibrinogen were determined in CatLab through nephelometry (Beckman Coulter Immage 800) and PT-derived fibrinogen ( $F B_{\mathrm{PT}}$ ) assay, respectively. Howbeit, fibrinogen levels of 29 adolescents were measured with STA-Neoplastin-Plus reactive (STA-Rack) while for the rest of participants was used Recombiplastin $2 \mathrm{G}$ reactive (ACLTOP 700). The inter-assay coefficient of variability for STARack was $6.52 \%$ while for ACLTOP 700 was $3.18 \%$. The intra-assay coefficient of variability for 
ACLTOP 700 was $0.75 \%$. Unfortunately, we cannot provide the coefficient of variability intraassay of STA-Rack because the register of its quality control is currently missing. TNF- $\alpha$ and IL- 6 concentrations were determined at the Research Center on Metabolism (Universitat de Barcelona). TNF- $\alpha$ was measured using a Quantikine HS ELISA Human TNF- $\alpha$ (R\&D Systems, Ref. HSTA00E Lot P174731) and IL-6 were determined by Quantikine HS ELISA Human IL-6 (R\&D Systems, Ref. HS600C Lot P178994). All these immunoassays were performed according to the manufacturer's instructions.

\section{BMI calculations}

$\mathrm{BMI}$, which results from dividing the weight in kilograms $(\mathrm{kg})$ by the square of height in meters $\left(\mathrm{m}^{2}\right)$, is the most common anthropometric measure used to determine obesity in adults [1]. Likewise, in pediatric population, the BMI is also used as a measure of adiposity since it is strongly correlated with body fat mass [41].

Healthy BMI range in childhood varies considerably with age. Hence, in order to achieve a comparable measurement of $\mathrm{BMI}$ among participants in our sample, we transformed $\mathrm{BMI}$ into BMI z-score (BMIz) using the 2000 CDC growth charts [31]. These guidelines allow the obtention of age and sex-specific BMI percentiles and BMIz. Although both are equivalent, we decided to use BMIz because it is more suitable for statistical analysis [42]. For the 21-year-old participants, their BMI was calculated as if they were 20 .

\section{Statistical analyzes}

Brain structure was assessed using two measures: cortical thickness and surface area. First, we explored the relationship between BMI, cortical thickness and surface area in a vertex-by-vertex fashion through Query Design Estimate Contrast (Qdec) interface of FreeSurfer. Age, gender (discrete variable) and years of education were set as nuisance factors in all image analysis to avoid its potential biasing effects over brain structure. In addition, total surface area was also controlled in surface area analysis. Analyzes were performed separately for each hemisphere with a $10 \mathrm{~mm}$ full-width at half maximum kernel. Results were corrected for multiple comparisons using a Monte-Carlo null-Z simulation (10,000 repetitions). Statistical significance was set at a cluster-wise corrected $p$-value $(C W P)<0.0125(0.05 / 4)$ after considering the analysis of both cortical metrics (i.e., thickness and surface area) over the two hemispheres. Significant clusters were reported according to the Desikan's atlas [39] in MNI305 space.

Second, we performed Spearman correlations between BMIz and the serum levels of each inflammatory agent obtained (i.e., IL-6, TNF- $\alpha$, CRP and fibrinogen) to ensure that these biomarkers were related with $\mathrm{BMI}$ in our sample.

Next, we addressed the putative link between inflammatory agents and the resulting neuroanatomical alterations originally linked to an increase in BMI. For this, cortical thickness and surface area values from significant and trending clusters were extracted for further posthoc analyzes in IBM SPSS Statistics (version 23). We used backward multiple linear regression models to explore the influence of all inflammatory parameters with each significant cluster. 
Here we used standardized residuals to control for the nuisance factors included in our previous vertex-wise analyzes. The significance threshold was set at Bonferroni-adjusted $p$-value $<0.05$. We also examined critical parameters of regression analysis to assure assumption fulfillment [43], which are available in the Supplementary Material section (Appendix A.2).

\section{RESULTS}

\section{Vertex-wise analyzes}

There was a negative relationship between cortical thickness $(\mathrm{mm})$ and BMIz in the left lateral occipital cortex (LOC) and the right precentral gyrus. Moreover, we have also identified a trend that negatively correlates the cortical thickness of the left fusiform gyrus with the increases in BMIz. Regarding surface area $\left(\mathrm{mm}^{2}\right)$, we found a positive relationship with BMIz in the left rostral middle frontal gyrus and the right superior frontal gyrus (Table 2; Figure 1-2). To assure the robustness and reliability of the results, we repeated the analyzes excluding the six 21-year-old participants. See Supplementary Material section (Appendix A.3).

\section{Correlation analyzes between BMIz and inflammatory biomarkers}

Spearman correlations reveled that BMlz was positively related with serum levels of hs-CRP $\left(r_{s}=\right.$ $0.568, p<0.001)$, IL-6 $\left(r_{s}=0.559, p<0.001\right)$, TNF- $\alpha\left(r_{s}=0.320, p=0.009\right)$ and fibrinogen $\left(r_{s}=0.514\right.$, $p<0.001)$. All of them survived Bonferroni adjustment. See Supplementary Material section (Appendix A.4). Correlations between inflammatory biomarkers are also included in Supplementary Material section (Appendix A.5).

\section{Post-hoc regression models of inflammation biomarkers}

Post-hoc regression analyzes showed that cortical thickness from significant and trending clusters associated with higher BMIz were partially explained by the increase in serum levels of fibrinogen in the left $\operatorname{LOC}\left(R^{2}=0.19, \beta=-0.45, p<0.001\right)$ and the left fusiform gyrus $\left(R^{2}=0.09\right.$, $\beta=-0.33, p=0.035)$. Contrarily, the right precentral gyrus thickness did not exhibit a significant relationship with fibrinogen values $(\beta=-0.24, p=0.057)$. Likewise, the mean surface within the right superior frontal gyrus were explained to some degree through serum levels of TNF- $\alpha\left(R^{2}=\right.$ $0.09, \beta=0.32, p=0.045$ ). In addition, although blood hs-CRP concentrations also partly explained surface increases in the left rostral middle frontal gyrus $\left(R^{2}=0.06, \beta=0.28, p=0.024\right)$, it did not pass the Bonferroni adjustment $(\beta=0.28, p=0.12)$. Backward regression models are presented in detail in Supplementary Material section (Appendix A.6). Best-fitting significant uncorrected regression models with adjusted p-value are shown in Table 3. Figure 3 shows the models that survived the multiple comparisons correction. 


\section{DISCUSSION}

This study aimed to assess the correlation between BMI and neuroanatomical changes in adolescents as well as the role that inflammatory agents might have over this relationship. We decided to use BMI instead of waist circumference or the waist-to-height ratio because BMI can be easily transformed into an age and sex-specific measure according to population rates (i.e., BMI z-score). None of the teenagers included in the study had any obesity-related comorbidity. This rigorous selection of participants was carried out with the aim of trying to isolate as best as possible the effect of BMI on brain structure, avoiding the potential confounding effects from other clinical comorbidities associated with unhealthy excess weight (e.g., insulin resistance, type 2 diabetes).

\section{Associations between cortical gray matter and BMI}

Using a vertex-wise approach, we found cortical thickness reductions associated with an increase in the BMIz in the left LOC, extending to the inferior parietal cortex and the right precentral gyrus, including the caudal middle frontal gyrus. In addition to that, we also found a trend that negatively correlates the cortical thickness of the left fusiform gyrus with the increases in BMIz. Our results are highly consistent with studies conducted in adults. Cortical thinning linked to BMI was also described in the left LOC [44], as well as in the left inferior parietal cortex, including LOC and fusiform areas, and the right precentral gyrus [45]. Both studies carried out vertex-wise correlations with a wide range of BMI that included healthy and unhealthy values that are compatible with obesity.

However, our results are not in line with other work exploring cortical thickness changes related to $\mathrm{BMI}$ in teenagers. To date, two studies did not find an association between BMI and cortical thickness $[15,16]$. The discrepancy between our results and these works could be explained by the age range of the sample, especially the upper age limit, because while both studies included adolescents not older than 18 , we included participants in late adolescence. Moreover, this inconsistency also might account either because differences in the sample size or the software employed.

Even though cortical areas in which we identified thickness changes related to weight increase have not been detected in adolescents so far, there is evidence to think that these brain regions could be altered in teenagers at an unhealthy weight situation, as they are involved in some aspects of feeding behavior. Both the LOC and the fusiform gyrus are two of the most reported areas in $\mathrm{fMRI}$ studies that investigate neural response to food stimuli [46]. Likewise, the LOC has also been linked to BMI in previous cortical thickness studies [44, 45], where it was typically related to visuospatial processing deficits in obesity. Additionally, in an electroencephalographic study, left LOC was pointed as one of several regions involved in salience tracking of high-energy and low-energy food and it was proposed to play a role in object recognition [47]. On the other hand, although precentral gyrus abnormalities are not too contrasted in unhealthy weight gain, this area has been described as a component of motor sensory networks implicated in visual recognition and control for food tasks [48]. 
Regarding surface area, we found a positive correlation with BMIz in two clusters with maximum peaks in the left rostral middle frontal gyrus, extending to the pars opercularis and the caudal middle frontal gyrus, and the right superior frontal gyrus, which included the caudal middle frontal, the rostral middle frontal and the precentral gyri. Both areas belong to the dorsolateral prefrontal cortex (DLPFC). As far as we know, only Saute and colleagues [16] examined the possible association between adolescents' surface area and BMI. They did not find a relationship between surface area and BMI in 44 teenagers from 15 to 18 .

The DLPFC is among the latest brain regions to mature [49]. In typical development, the cortical area of the DLPFC shows age-related declines from 9 to 20 years old [50]. Accordingly, an increase in DLPFC surface area could be interpreted as an interference of the BMI-related inflammation with the prefrontal cortex synaptic pruning process. This mechanism could potentially reduce the effectiveness of frontal lobe functions in adolescence and later life. This speculation may be in line with the strong evidence that supports the existence of a negative relationship between executive functioning and unhealthy weight in teenagers [51, 52]. Nevertheless, smaller surface area in prefrontal regions has been associated with problems in executive functioning in adults [11]. Clearly, more studies are needed to validate these results.

Another plausible explanation would be to consider that unhealthy BMI-increase may also be affecting underlying white matter, since tension-changes along the axons of white matter has been described as the primary driving force for cortical folding [53]. Likewise, a magnetoencephalographic study conducted in young females showed an increased resting-state functional connectivity in obese adolescents [54]. The authors pointed to the white matter expansion as a possible cause of this altered pattern of connectivity, suggesting that white matter expansion in obese people could be a reflection of abnormal lipid metabolism and increased accumulation of fat in central myelin throughout the brain. Therefore, changes in the DLPFC area could also be an indirect consequence of possible white matter alterations.

\section{Cortical gray matter findings and proinflammatory agents}

Addressing another main objective, we found that the relationship between gray matter structure and BMI was partly explained by inflammatory agents. Higher fibrinogen serum concentrations were related to thinning within the left LOC and the left fusiform gyrus. Alternatively, higher serum levels of TNF- $\alpha$ were associated to a greater surface area in the right superior frontal gyrus.

Although the relationship between obesity and low-grade chronic inflammation is well known, our results suggest that peripheral inflammatory-related agents are associated with brain structure. Several studies have reported a significant correlation between the levels of cytokines in blood and in the cerebrospinal fluid [29]. Thus, it could be possible that elevated serum levels of inflammatory biomarkers might be reflecting the existence of a neuroinflammatory state, which in turn would be involved in the cortical brain alterations that we have identified. The reason why fibrinogen and TNF- $\alpha$ are related to opposite patterns (i.e., decrease and increase) and different metrics (i.e., thickness and surface) remains unclear. Two hypothetical explanations could be that (1) the course of the neuroinflammatory response may be 
disharmonious among different brain regions, and (2) the effect of neuroinflammation could be subjected to the specific developmental stage of each cortical region.

Recent revisions show evidence that diet-induced obesity compromises the integrity of bloodbrain barrier (BBB) through endothelial dysfunction $[19,55,56]$. Although the most reported evidence is based in animal models of obesity, Gustafson et al. (2007) suggested that BBB permeability is also higher in overweight or obese people than controls [56]. Reduction in cortical thickness has been interpreted as a proxy of atrophy [57], as it might mirror neuronal loss, reduced size of neural cell bodies or degradation [10]. The brain regions we have described as thinner in relation to the $\mathrm{BMI}$ increase were those exhibiting a link with fibrinogen. Under normal circumstances, serum fibrinogen cannot penetrate into the central nervous system (CNS) due to the BBB. However, when the BBB is disrupted fibrinogen could leak from blood vessels and deposit in the CNS [58]. Although we have no evidence of the presence of fibrinogen in the CNS of our participants, the fact that fibrinogen was the serum inflammatory agent more associated with cortical thickness reductions could point towards that the inflammatory response might be at a more advanced stage. Likewise, other studies have demonstrated that fibrinogen is related with both neural death and synaptic degradation [59] as well as with reductions in neural density [60]; findings that could fit with a reduction in cortical thickness. Conversely, the fact that the changes related to BMI in the area of DLPFC were related to TNF- $\alpha$ but not to fibrinogen could mean that the neuroinflammatory response within DLPFC might be at an earlier stage, still incapable of causing a decrease in gray matter. However, after some time, the course of this response would continue advancing and would be able to produce gray matter decreases. This idea would be congruent with results reported by Cazettes and colleagues [30], since they found that the lateral orbitofrontal cortex volumes of overweight/obese adults were negatively associated with fibrinogen.

As above mentioned, all regions of the brain do not end their development at the same time, being the prefrontal cortex the last brain region to reach adult maturity [49]. Hence, it might be possible that potential neuroinflammation response related to BMI could exercise different effects in different cortical regions as well as make different brain regions more susceptible to certain inflammatory biomarkers depending on the specific moment of development they are going through. Finally, it is worth considering that both possible interpretations (i.e., the desynchronized course of the neuroinflammatory response and the specific developmental stage of each brain region) might be complementary rather than exclusive.

However, it is necessary to highlight again that these are only hypothetical explanations. On one side, there is no evidence that the permeability of BBB is disrupted in our sample. Moreover, it is worth noting that we only measured proinflammatory agents in serum.

\section{LIMITATIONS AND FUTURE DIRECTIONS}

This study has some limitations that should be acknowledged. Parametric cluster-wise MonteCarlo null-Z simulation analyzes could potentially overestimate some of our results. For this reason, we additionally performed a strict permutation testing approach. While this additional analysis showed similar results in cortical thickness, results in surface area did not reach 
significance. While it is true that our sample size could have not enough statistical power to detect subtle cortical differences across healthy adolescents who only differ in BMI in permutation testing, there is not a validated method to calculate sample size in structural MRI studies. In addition, there are also methodological limitations that are worth to be pointed. The 2000 CDC growth charts [31] only allow to calculate BMIz for individuals up to 20 years. Our study, however, included six 21-year-old participants to cover the entire period of adolescence. For this reason, we repeated the analyzes excluding the six 21-year-old participants, finding similar relationships between $\mathrm{BMI}$, cortical thickness and area, albeit, as predictably, with a decreased corrected p-value (Appendix A.3). Besides that, despite some evidence towards a correlation between the levels of cytokines in the blood and in the cerebrospinal fluid [29], any interpretation about the concentration of fibrinogen and cytokines in the CNS is utterly speculative. Hence, although we found an association between systemic inflammation obesityrelated and brain structure, we cannot conclude that obesity is linked to a neuroinflammation state that alters the cortical mantle. Finally, the cross-sectional design of this study unfortunately does not allow us to conclude on causality. Therefore, as it is premature to draw firm conclusions, future research should continue the study of the possible relationship between cortical changes associated to BMI and inflammatory agents in larger samples. The confirmation of this association could help in the development of targeted interventions aimed at reducing peripheral obesity-related inflammation as well as its possible impact on the neuroinflammatory state and its harmful effects on the brain.

\section{CONCLUSIONS}

We have found a relationship between BMI and brain structure in adolescents. Specifically, we have detected that body mass increases were associated with thinner cortices in the left LOC and the right precentral gyrus as well as with greater surface area in the left rostral middle frontal gyrus and the right superior frontal gyrus. Likewise, we have also identified a trend between thickness decreases in the left fusiform gyrus and body mass increases. All these areas could play a relevant role in some aspects of feeding behavior. Moreover, we have evidenced that these cortical changes were partially associated with peripheral inflammatory agents such as fibrinogen and TNF- $\alpha$.

\section{ACKNOWLEDGMENTS}

The authors thank all participants in the study without whose support the work would not have been possible.

\section{Funding}

This work was supported by grants from MINECO to Dr. Maria Angeles Jurado (PSI2017-86536C2-1-R) and Dr. Maite Garolera (PSI2017-86536-C2-2-R) and from the Generalitat de Catalunya to Xavier Prats-Soteras (FI-DGR 2017). 


\section{Author contributions}

XPS, MAJ, JOG, IGG, BS, XC, and MG contributed to study design and conception, analyzes and results interpretation. XPS, JOG, IGG, CSG, NM, CT and MSP participated in data acquisition. Additionally, all authors critically revisited the work, approved its final version for publishing, and agreed to be accountable for all aspects of such work.

\section{CONFLICT OF INTEREST}

The authors declare that they have no conflict of interest. 


\section{REFERENCES}

1. WHO. (2018). Obesity and overweight. Retrieved January 23,2019 , from https://www.who.int/news-room/fact-sheets/detail/obesity-and-overweight

2. Reilly, J. J., \& Kelly, J. (2011). Long-term impact of overweight and obesity in childhood and adolescence on morbidity and premature mortality in adulthood: systematic review. International Journal of Obesity, 35(7), 891-898. https://doi.org/10.1038/ijo.2010.222

3. García-García, I., Michaud, A., Dadar, M., Zeighami, Y., Neseliler, S., Collins, D. L., et al. (2018). Neuroanatomical differences in obesity: meta-analytic findings and their validation in an independent dataset. International Journal of Obesity, 9. https://doi.org/10.1038/s41366-018-0164-4

4. Alosco, M. L., Stanek, K. M., Galioto, R., Korgaonkar, M. S., Grieve, S. M., Brickman, A. M., et al. (2014). Body mass index and brain structure in healthy children and adolescents. International Journal of Neuroscience, 124(1), 49-55. https://doi.org/10.3109/00207454.2013.817408

5. Kennedy, J. T., Collins, P. F., \& Luciana, M. (2016). Higher adolescent body mass index is associated with lower regional gray and white matter volumes and lower levels of positive emotionality. Frontiers in Neuroscience, 10(SEP), 1-12. https://doi.org/10.3389/fnins.2016.00413

6. Vijayakumar, N., Allen, N. B., Youssef, G., Dennison, M., Yücel, M., Simmons, J. G., et al. (2016). Brain development during adolescence: A mixed-longitudinal investigation of cortical thickness, surface area, and volume. Human Brain Mapping, 37(6), 2027-2038. https://doi.org/10.1002/hbm.23154

7. Winkler, A. M., Greve, D. N., Bjuland, K. J., Nichols, T. E., Sabuncu, M. R., Håberg, A. K., et al. (2018). Joint Analysis of Cortical Area and Thickness as a Replacement for the Analysis of the Volume of the Cerebral Cortex. Cerebral Cortex, 28(2), 738-749. https://doi.org/10.1093/cercor/bhx308

8. Rimol, L. M., Nesvåg, R., Hagler, D. J., Bergmann, $\varnothing$., Fennema-Notestine, C., Hartberg, C. B., et al. (2012). Cortical Volume, Surface Area, and Thickness in Schizophrenia and Bipolar Disorder. https://doi.org/10.1016/j.biopsych.2011.11.026

9. Natu, V. S., Gomez, J., Barnett, M., Jeska, B., Kirilina, E., Jaeger, C., et al. (2019). Apparent thinning of human visual cortex during childhood is associated with myelination. Proceedings of the National Academy of Sciences of the United States of America, 116(41), 20750-20759. https://doi.org/10.1073/pnas.1904931116

10. Worker, A., Blain, C., Jarosz, J., Chaudhuri, K. R., Barker, G. J., Williams, S. C. R., et al. (2014). Cortical thickness, surface area and volume measures in Parkinson's disease, multiple system atrophy and progressive supranuclear palsy. PloS One, 9(12), e114167. https://doi.org/10.1371/journal.pone.0114167

11. Tamnes, C. K., Zeller, B., Amlien, I. K., Kanellopoulos, A., Andersson, S., Due-Tønnessen, P., et al. (2015). Cortical surface area and thickness in adult survivors of pediatric acute lymphoblastic leukemia. Pediatric Blood \& Cancer, 62(6), 1027-1034. https://doi.org/10.1002/pbc.25386

12. Ross, N., Yau, P. L., \& Convit, A. (2015). Obesity, fitness, and brain integrity in adolescence. Appetite. https://doi.org/10.1016/j.appet.2015.03.033

13. Yau, P. L., Kang, E. H., Javier, D. C., \& Convit, A. (2014). Preliminary evidence of cognitive and brain abnormalities in uncomplicated adolescent obesity. Obesity, 00(00), n/a-n/a. https://doi.org/10.1002/oby.20801

14. de Groot, C. J., van den Akker, E. L. T., Rings, E. H. H. M., Delemarre-van de Waal, H. A., \& van der Grond, J. (2017). Brain structure, executive function and appetitive traits in 
adolescent obesity. Pediatric Obesity, 12(4), e33-e36.

https://doi.org/10.1111/ijpo.12149

15. Sharkey, R. J., Karama, S., \& Dagher, A. (2015). Overweight is not associated with cortical thickness alterations in children. Frontiers in Neuroscience, 9(February), 1-7. https://doi.org/10.3389/fnins.2015.00024

16. Saute, R. L., Soder, R. B., Alves Filho, J. O., Baldisserotto, M., \& Franco, A. R. (2018). Increased brain cortical thickness associated with visceral fat in adolescents. Pediatric Obesity, 13(1), 74-77. https://doi.org/10.1111/ijpo.12190

17. Westwater, M. L., Vilar-López, R., Ziauddeen, H., Verdejo-García, A., \& Fletcher, P. C. (2019). Combined effects of age and BMI are related to altered cortical thickness in adolescence and adulthood. Developmental Cognitive Neuroscience, 40(November). https://doi.org/10.1016/i.dcn.2019.100728

18. Ronan, L., Alexander-Bloch, A., \& Fletcher, P. C. (2019). Childhood Obesity, Cortical Structure, and Executive Function in Healthy Children. Cerebral Cortex, 1-10. https://doi.org/10.1093/cercor/bhz257

19. Guillemot-Legris, O., \& Muccioli, G. G. (2017). Obesity-Induced Neuroinflammation: Beyond the Hypothalamus. Trends in Neurosciences, 40(4), 237-253. https://doi.org/10.1016/i.tins.2017.02.005

20. Reilly, S. M., \& Saltiel, A. R. (2017). Adapting to obesity with adipose tissue inflammation. Nature Reviews Endocrinology, 13(11), 633-643. https://doi.org/10.1038/nrendo.2017.90

21. Kim, Y. K., \& Won, E. (2017). The influence of stress on neuroinflammation and alterations in brain structure and function in major depressive disorder. Behavioural Brain Research, 329(April), 6-11. https://doi.org/10.1016/j.bbr.2017.04.020

22. Kang, M., Vaughan, R. A., \& Paton, C. M. (2015). FDP-E induces adipocyte inflammation and suppresses insulin-stimulated glucose disposal: effect of inflammation and obesity on fibrinogen B $\beta$ mRNA. American Journal of Physiology-Cell Physiology, 309(11), C767-C774. https://doi.org/10.1152/ajpcell.00101.2015

23. Miller, A. L., Lee, H. J., \& Lumeng, J. C. (2015). Obesity-associated biomarkers and executive function in children. Pediatric Research, 77(1-2), 143-147. https://doi.org/10.1038/pr.2014.158

24. Nguyen, J. C. D., Killcross, A. S., \& Jenkins, T. A. (2014). Obesity and cognitive decline: role of inflammation and vascular changes. Frontiers in Neuroscience, 8(OCT), 1-9. https://doi.org/10.3389/fnins.2014.00375

25. Vachharajani, V., \& Granger, D. N. (2009). Adipose tissue: A motor for the inflammation associated with obesity. IUBMB Life, 61(4), 424-430. https://doi.org/10.1002/iub.169

26. Chow, B. W., \& Gu, C. (2015). The Molecular Constituents of the Blood-Brain Barrier. Trends in Neurosciences, 38(10), 598-608. https://doi.org/10.1016/j.tins.2015.08.003

27. Miller, A. H., Haroon, E., Raison, C. L., \& Felger, J. C. (2013). Cytokine targets in the brain: Impact on neurotransmitters and neurocircuits. Depression and Anxiety, 30(4), 297-306. https://doi.org/10.1002/da.22084

28. Ryu, J. K., \& McLarnon, J. G. (2009). A leaky blood-brain barrier, fibrinogen infiltration and microglial reactivity in inflamed Alzheimer's disease brain. Journal of Cellular and Molecular Medicine, 13(9 A), 2911-2925. https://doi.org/10.1111/i.15824934.2008.00434.x

29. Rosano, C., Marsland, A. L., \& Gianaros, P. J. (2012). Maintaining brain health by monitoring inflammatory processes: A mechanism to promote successful aging. Aging and Disease, 3(1), 16-33.

30. Cazettes, F., Cohen, J. I., Yau, P. L., Talbot, H., \& Convit, A. (2011). Obesity-mediated inflammation may damage the brain circuit that regulates food intake. Brain Research, 1373, 101-109. https://doi.org/10.1016/i.brainres.2010.12.008 
31. Kuczmarski, R. J., Ogden, C. L., Guo, S. S., Grummer-Strawn, L. M., Flegal, K. M., Mei, Z., et al. (2002). 2000 CDC Growth Charts for the United States: methods and development. Vital and Health Statistics. Series 11, Data from the National Health Survey, (246), 1-190. Retrieved from http://www.ncbi.nlm.nih.gov/pubmed/12043359

32. Alberti, K. G. M. M., Eckel, R. H., Grundy, S. M., Zimmet, P. Z., Cleeman, J. I., Donato, K. A., et al. (2009). Harmonizing the Metabolic Syndrome. Circulation, 120(16), 16401645. https://doi.org/10.1161/CIRCULATIONAHA.109.192644

33. Yau, P. L., Castro, M. G., Tagani, A., Tsui, W. H., \& Convit, A. (2012). Obesity and metabolic syndrome and functional and structural brain impairments in adolescence. Pediatrics, 130, e856-64. https://doi.org/10.1542/peds.2012-0324

34. Wechsler, D. (2007). WISC-IV: Escala de Inteligencia de Wechsler para Niños-IV (2a ed.). Madrid: TEA.

35. Wechsler, D. (1999). WAIS III. Escala de Inteligencia de Wechsler para Adultos III (Adaptación espa-ola ed.). Madrid: TEA Editores, S.A.

36. Reuter, M., Rosas, H. D., \& Fischl, B. (2010). Highly accurate inverse consistent registration: A robust approach. Neurolmage, 53(4), 1181-1196.

https://doi.org/10.1016/j.neuroimage.2010.07.020

37. Ségonne, F., Dale, A. M., Busa, E., Glessner, M., Salat, D., Hahn, H. K., et al. (2004). A hybrid approach to the skull stripping problem in MRI. Neurolmage, 22(3), 1060-1075. https://doi.org/10.1016/i.neuroimage.2004.03.032

38. Sled, J. G., Zijdenbos, A. P., \& Evans, A. C. (1998). A nonparametric method for automatic correction of intensity nonuniformity in MRI data. IEEE Transactions on Medical Imaging, 17(1), 87-97. https://doi.org/10.1109/42.668698

39. Desikan, R. S., Ségonne, F., Fischl, B., Quinn, B. T., Dickerson, B. C., Blacker, D., et al. (2006). An automated labeling system for subdividing the human cerebral cortex on MRI scans into gyral based regions of interest. Neurolmage, 31(3), 968-980. https://doi.org/10.1016/j.neuroimage.2006.01.021

40. Fischl, B., \& Dale, A. M. (2000). Measuring the thickness of the human cerebral cortex from magnetic resonance images. Proceedings of the National Academy of Sciences, 97(20), 11050-11055. https://doi.org/10.1073/pnas.200033797

41. Martin-Calvo, N., Moreno-Galarraga, L., \& Martinez-Gonzalez, M. A. (2016). Association between Body Mass Index, Waist-to-Height Ratio and Adiposity in Children: A Systematic Review and Meta-Analysis. https://doi.org/10.3390/nu8080512

42. Must, A., \& Anderson, S. E. (2006). PEDIATRIC MINI REVIEW Body mass index in children and adolescents: considerations for population-based applications. International Journal of Obesity, 30, 590-594. https://doi.org/10.1038/sj.ijo.0803300

43. Field, A. P. (2005). Discovering statistics using SPSS :(and sex, drugs and rock'n' roll) (Vol. 2). London etc.: Sage Publications.

44. Medic, N., Ziauddeen, H., Ersche, K. D., Farooqi, I. S., Bullmore, E. T., Nathan, P. J., et al. (2016). Increased body mass index is associated with specific regional alterations in brain structure. International Journal of Obesity, 40(7), 1177-1182. https://doi.org/10.1038/ijo.2016.42

45. Veit, R., Kullmann, S., Heni, M., Machann, J., Häring, H.-U., Fritsche, A., et al. (2014). Reduced cortical thickness associated with visceral fat and BMI. Neurolmage: Clinical, 6, 307-311. https://doi.org/10.1016/i.nicl.2014.09.013

46. van der Laan, L. N., de Ridder, D. T., Viergever, M. A., \& Smeets, P. A. (2011). The first taste is always with the eyes: a meta-analysis on the neural correlates of processing visual food cues. Neurolmage, 55(1), 296-303. https://doi.org/10.1016/j.neuroimage.2010.11.055

47. Toepel, U., Knebel, J.-F., Hudry, J., Le Coutre, J., \& Murray, M. M. (2008). The brain tracks the energetic value in food images. Neurolmage, 44, 967-974.

https://doi.org/10.1016/i.neuroimage.2008.10.005 
48. Kullmann, S., Pape, A.-A., Heni, M., Ketterer, C., Schick, F., Häring, H.-U., et al. (2013). Functional network connectivity underlying food processing: disturbed salience and visual processing in overweight and obese adults. Cerebral Cortex (New York, N.Y. : 1991), 23(5), 1247-1256. https://doi.org/10.1093/cercor/bhs124

49. Giedd, J. N. (2004). Structural Magnetic Resonance Imaging of the Adolescent Brain. Annals of the New York Academy of Sciences, 1021(1), 77-85. https://doi.org/10.1196/annals.1308.009

50. Mensen, V. T., Wierenga, L. M., van Dijk, S., Rijks, Y., Oranje, B., Mandl, R. C. W., et al. (2017). Development of cortical thickness and surface area in autism spectrum disorder. Neurolmage. Clinical, 13, 215-222. https://doi.org/10.1016/j.nicl.2016.12.003

51. Liang, J., Matheson, B. E., Kaye, W. H., \& Boutelle, K. N. (2014). Neurocognitive correlates of obesity and obesity-related behaviors in children and adolescents. International Journal of Obesity, 38(4), 494-506. https://doi.org/10.1038/ijo.2013.142

52. Reinert, K. R. S., Po'e, E. K., \& Barkin, S. L. (2013). The Relationship between Executive Function and Obesity in Children and Adolescents: A Systematic Literature Review. Journal of Obesity, 2013(2), 1-10. https://doi.org/10.1155/2013/820956

53. Essen, D. (1997). A tension-based theory of morphogenesis and compact wiring in the central nervous system. Nature, 385(6614), 313-318. https://doi.org/10.1038/385313a0

54. Olde Dubbelink, K. T. E., Felius, A., Verbunt, J. P. A., van Dijk, B. W., Berendse, H. W., Stam, C. J., et al. (2008). Increased resting-state functional connectivity in obese adolescents; A magnetoencephalographic pilot study. PLOS ONE, 3(7), 1-6. https://doi.org/10.1371/journal.pone.0002827

55. Buie, J. J., Watson, L. S., Smith, C. J., \& Sims-Robinson, C. (2019). Obesity-related cognitive impairment: The role of endothelial dysfunction. Neurobiology of Disease, 132(March). https://doi.org/10.1016/j.nbd.2019.104580

56. Chan, K. L., Cathomas, F., \& Russo, S. J. (2019). Central and peripheral inflammation link metabolic syndrome and major depressive disorder. Physiology, 34(2), 123-133. https://doi.org/10.1152/physiol.00047.2018

57. Hutton, C., Draganski, B., Ashburner, J., \& Weiskopf, N. (2009). A comparison between voxel-based cortical thickness and voxel-based morphometry in normal aging. Neurolmage, 48(2), 371-380. https://doi.org/10.1016/j.neuroimage.2009.06.043

58. Liu, Y., Chen, H., Zhao, K., He, W., Lin, S., \& He, J. (2019). High levels of plasma fibrinogen are related to post-stroke cognitive impairment. Brain and Behavior, $9(10)$. https://doi.org/10.1002/brb3.1391

59. Cortes-Canteli, M., Mattei, L., Richards, A. T., Norris, E. H., \& Strickland, S. (2015). Fibrin deposited in the Alzheimer's disease brain promotes neuronal degeneration. Neurobiology of Aging, 36(2), 608-617. https://doi.org/10.1016/j.neurobiolaging.2014.10.030

60. Jenkins, D. R., Craner, M. J., Esiri, M. M., \& Deluca, G. C. (2018). Contribution of Fibrinogen to Inflammation and Neuronal Density in Human Traumatic Brain Injury. Journal of Neurotrauma, 35(19), 2259-2271. https://doi.org/10.1089/neu.2017.5291 


\section{Table 1}

Demographic, anthropometric and inflammatory characteristics.

$B M I z=$ body mass index $z$-score $; H W=$ healthy weight; $O W=$ overweight $O=$ obese $F=$ female;

\begin{tabular}{lccc}
\hline & Mean & SD & Range \\
\hline Age (years) & 15.89 & 2.72 & $12-21$ \\
BMIz & 1.07 & 1.06 & $-1.28-2.69$ \\
Gender (F/M) & \multicolumn{2}{c}{$33 / 32$} \\
Weight status (HW/OW/O) & \multicolumn{2}{c}{$28 / 10 / 27$} & \\
Years education & 9.74 & 2.38 & $6-16$ \\
IQ estimation (scalar score) & 11.17 & 2.22 & $7-17$ \\
hs-CRP (mg/dL) & 3.45 & 9.11 & $0.12-67.80$ \\
TNF- $\alpha$ (pg/mL) & 0.89 & 0.35 & $0.26-1.95$ \\
IL-6 (pg/mL) & 2.41 & 3.11 & $0.38-18.76$ \\
Fibrinogen ${ }^{\text {a }}$ (g/L) & 3.67 & 0.81 & $2.23-6.04$ \\
Fibrinogen STA-Rack (g/L) & 3.67 & 0.66 & $2.60-5.33$ \\
Fibrinogen ACLTOP 700 (g/L) & 3.67 & 0.91 & $2.23-6.04$ \\
\hline
\end{tabular}

$M=$ male; $I Q$ estimation = Intelligence Quotient estimation; $h s-C R P=$ high-sensitivity C-reactive protein; $\mathrm{mg} / \mathrm{dL}=$ milligrams per deciliter; $T N F-\alpha=$ tumor necrosis factor alpha; $\mathrm{pg} / \mathrm{mL}=$ picograms per milliliter; $I L-6=$ Interleukin-6; $g / L=$ grams per liter .

${ }^{a}$ Serum levels of fibrinogen considering all participants and both assay types (i.e., STA-Rack and ACLTOP 700).

Table 2

Vertex-wise results.

\begin{tabular}{|c|c|c|c|c|c|c|}
\hline \multirow[t]{2}{*}{ Peak location } & \multirow{2}{*}{$\begin{array}{l}\text { Cluster size } \\
\qquad\left(\mathrm{mm}^{2}\right)\end{array}$} & \multicolumn{3}{|c|}{ Peak MNI coordinates } & \multirow{2}{*}{$\begin{array}{l}\text { Statistical } \\
\text { (Z) }\end{array}$} & \multirow{2}{*}{$\begin{array}{c}\text { Corrected } \\
\text { p-value }\end{array}$} \\
\hline & & $x$ & Y & Z & & \\
\hline \multicolumn{7}{|l|}{ Thickness } \\
\hline L-LOC & 958.80 & -41.6 & -78.8 & -2.5 & -2.756 & 0.0092 \\
\hline L-Fusiform & 885.96 & -32.9 & -38.3 & -17.8 & -3.166 & 0.0177 \\
\hline R-Precentral & 1748.90 & 22.7 & -19.0 & 55.2 & -3.507 & 0.0001 \\
\hline \multicolumn{7}{|l|}{ Surface area } \\
\hline L-Rostral middle frontal & 2528.97 & -34.9 & 29.5 & 31.7 & 3.29 & 0.0014 \\
\hline R-Superior frontal & 2930.23 & 7.3 & 31.8 & 44.1 & 2.646 & 0.0008 \\
\hline
\end{tabular}

$L=$ left; $R=$ right; $L O C=$ lateral occipital cortex. 



\section{LH}

Surface area

$\mathrm{RH}$
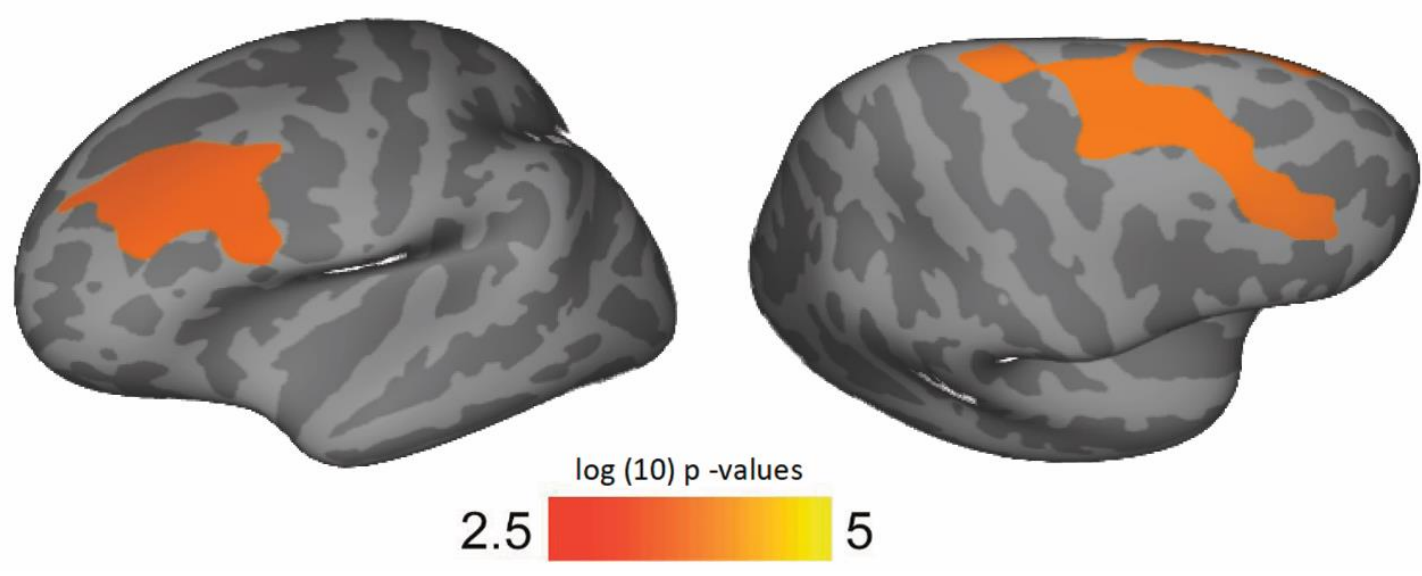

Figure 1. Lateral and inferior views of the thickness reduction in relation to BMIz after adjusting for age, gender and years of education. Significant clusters in the left LOC and the right precentral gyrus and trending cluster in the left fusiform gyrus (a). Lateral and superior views of increasing surface area in relation to the BMIz after adjusting for age, gender, years of education and total surface area. Significant clusters in the left rostral middle frontal gyrus and the right superior frontal gyrus (b). Scatter plots are shown in Figure 2. 

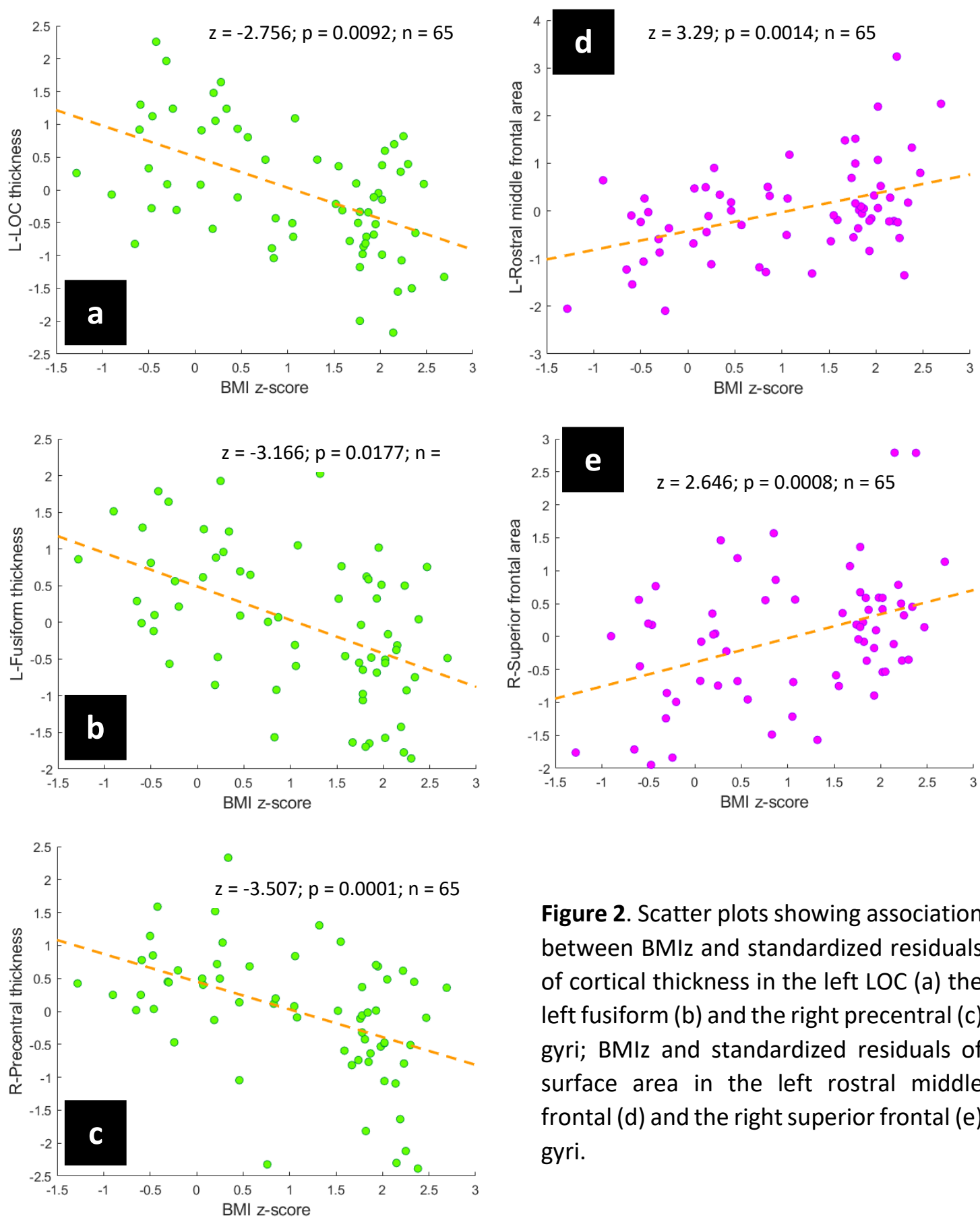

Figure 2. Scatter plots showing association between BMIz and standardized residuals of cortical thickness in the left LOC (a) the left fusiform (b) and the right precentral (c) gyri; BMIz and standardized residuals of surface area in the left rostral middle frontal (d) and the right superior frontal (e) gyri. 
Table 3.

Best-fitting significant uncorrected regression models with adjusted $p$-value.

\begin{tabular}{|c|c|c|c|c|c|c|c|}
\hline & Biomarker & ${ }^{+} \mathrm{R}^{2}$ & $\beta$ & $\mathrm{t}$ & SE & $p$ & $\begin{array}{c}\text { Bonferroni } \\
\text { adjusted } \\
\text { p-value }\end{array}$ \\
\hline \multicolumn{8}{|l|}{ Thickness } \\
\hline L-LOC & Fibrinogen & 0.19 & -0.45 & -3.99 & 0.14 & $<0.001 * * *$ & $<0.001 * * *$ \\
\hline L-Fusiform & Fibrinogen & 0.09 & -0.33 & -2.77 & 0.14 & $0.007 * *$ & $0.035^{*}$ \\
\hline \multicolumn{8}{|l|}{ Surface area } \\
\hline L-Ros mid frontal & hs-CRP & 0.06 & 0.28 & 2.31 & 0.01 & $0.024^{*}$ & 0.12 \\
\hline $\mathrm{R}$-Superior frontal & TNF- $\alpha$ & 0.09 & 0.32 & 2.70 & 0.33 & $0.009 * *$ & $0.045^{*}$ \\
\hline
\end{tabular}

${ }^{+}$Adjusted $R$ squared value; $S E=$ standard error; $L=$ left; $R=$ right; $L O C=$ lateral occipital cortex; Ros = rostral; mid = middle; TNF- $\alpha=$ tumor necrosis factor alpha; hs-CRP = high-sensitivity $C$ reactive protein; * $p<0.05$; $^{* *} p<0.01 ;{ }^{* * *} p<0.001$.

L-Fusiform: $\beta=-0.33 ; p=0.035$

L-Lateral occipital: $\beta=-0.45 ; p<0.001$

$\beta=0.32 ; p=0.045 ; n=65$
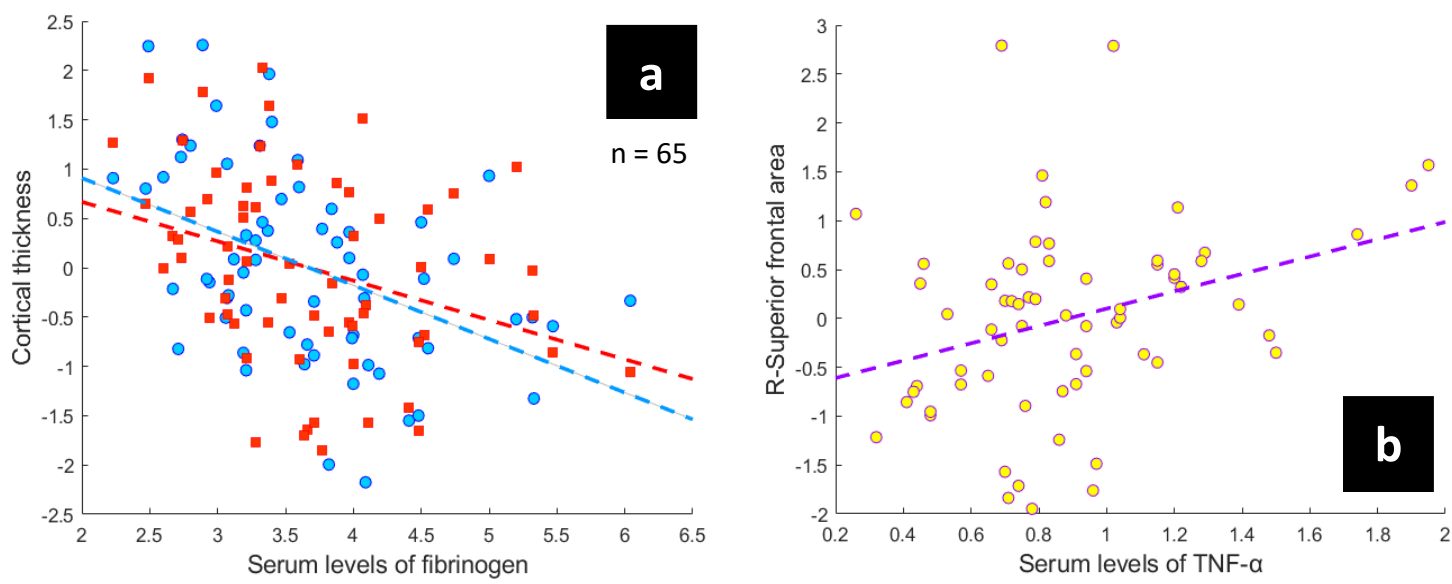

Figure 3. Scatter plots showing the association between standardized residuals of cortical thickness and blood fibrinogen concentration (a) and the link between standardized residuals of surface area and serum levels of TNF- $\alpha$ (b). 


\section{SUPPLEMENTARY MATERIAL}

Appendix A.1 - Flow of included and excluded participants in the study.

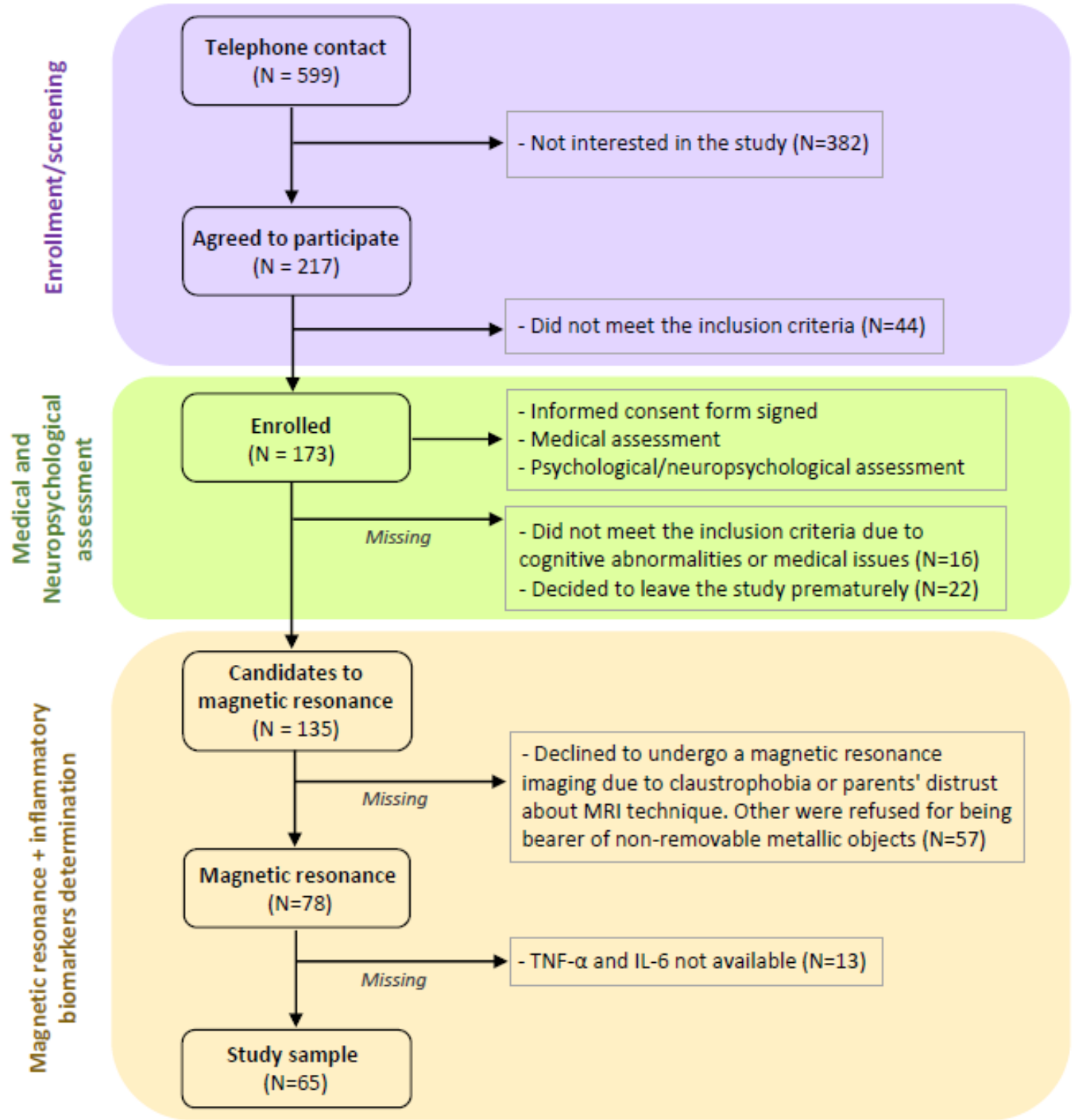


Appendix A.2 - Examined standardized residuals in regression models of inflammation biomarkers

\begin{tabular}{|c|c|c|c|c|c|}
\hline CODE & $\begin{array}{l}\text { Standardized } \\
\text { Residual }\end{array}$ & $\begin{array}{l}\text { Cook's } \\
\text { Distance }\end{array}$ & $\begin{array}{c}\text { Centered } \\
\text { Leverage Value }\end{array}$ & $\begin{array}{c}\text { Mahalanobis } \\
\text { Distance }\end{array}$ & $\begin{array}{l}\text { Covariance } \\
\text { Ratio (CVR) }\end{array}$ \\
\hline \multicolumn{6}{|l|}{ L-LOC } \\
\hline OI064 & -2.21561 & .05003 & .00421 & .26928 & .89240 \\
\hline OI017 & -2.17765 & .03896 & .00053 & .03382 & .89485 \\
\hline $\mathrm{Cl055}$ & 2.08438 & .06948 & .01470 & .94106 & .91855 \\
\hline $\mathrm{ClO65}$ & 2.05370 & .03808 & .00205 & .13110 & .91252 \\
\hline \multicolumn{6}{|c|}{ L-Fusiform thickness } \\
\hline OI045 & -2.07634 & .04274 & .00369 & .23635 & .91086 \\
\hline SI032 & 2.03314 & .03901 & .00281 & .17987 & .91579 \\
\hline \multicolumn{6}{|c|}{ R-Superior frontal area } \\
\hline $\mathrm{ClO68}$ & -2.00486 & .03502 & .00146 & .09347 & .91833 \\
\hline OI067 & 3.20944 & .10903 & .00493 & .31579 & .73150 \\
\hline OI049 & 2.88932 & .07617 & .00223 & .14255 & .78661 \\
\hline
\end{tabular}

$L=$ left; $R=$ right; $L O C=$ lateral occipital cortex.

Appendix A.3 - Vertex-wise results excluding the six 21-year-old participants.

\begin{tabular}{|c|c|c|c|c|c|c|}
\hline \multirow[t]{2}{*}{ Peak location } & \multirow{2}{*}{$\begin{array}{l}\text { Cluster size } \\
\qquad\left(\mathrm{mm}^{2}\right)\end{array}$} & \multicolumn{3}{|c|}{ Peak MNI coordinates } & \multirow{2}{*}{$\begin{array}{l}\text { Statistical } \\
\text { (Z) }\end{array}$} & \multirow{2}{*}{$\begin{array}{c}\text { Corrected } \\
\text { p-value }\end{array}$} \\
\hline & & $x$ & Y & Z & & \\
\hline \multicolumn{7}{|l|}{ Thickness } \\
\hline L-LOC & 822.39 & -43.3 & -69.0 & -2.0 & -2.536 & 0.0287 \\
\hline L-Fusiform & 778.57 & -32.3 & -38.7 & -17.5 & -3.135 & 0.0386 \\
\hline R-Precentral & 1319.42 & 23.4 & -18.7 & 55.3 & -3.103 & 0.0018 \\
\hline \multicolumn{7}{|l|}{ Surface area } \\
\hline L-Rostral middle frontal & 2743.18 & -35.5 & 29.2 & 31.4 & 3.129 & 0.0005 \\
\hline L-Superior frontal & 1631.18 & -13.2 & 32.8 & 18.2 & 2.947 & 0.0446 \\
\hline R-Superior frontal & 3713.02 & 7.1 & 33.1 & 43.8 & 2.926 & 0.0001 \\
\hline
\end{tabular}


Appendix A.4 - Spearman correlations between BMIz and inflammatory biomarkers with adjusted $p$-value.

\begin{tabular}{llc}
\hline Rho de Spearman & \\
\hline \multirow{4}{*}{ hs-CRP } & Correlation coefficient & BMIz \\
\cline { 2 - 2 } IL-6 & Sig (bilateral) & 0.568 \\
& Correlation coefficient & $<0.001^{* * *}$ \\
TNF- $\alpha$ & Sig (bilateral) & 0.559 \\
& Correlation coefficient & $<0.001^{* * *}$ \\
Fibrinogen & Sig (bilateral) & 0.320 \\
& Correlation coefficient & $0.009^{* *}$ \\
\hline Bonferroni adjusted p-value & 0.514 \\
\hline \multirow{4}{*}{ hs-CRP } & Sig (bilateral) & $<0.001^{* * *}$ \\
& Correlation coefficient & \\
& Sig (bilateral) & BMlz \\
IL-6 & Correlation coefficient & 0.568 \\
& Sig (bilateral) & $<0.001^{* * *}$ \\
TNF- $\alpha$ & Correlation coefficient & 0.559 \\
& Sig (bilateral) & $<0.001^{* * *}$ \\
Fibrinogen & Correlation coefficient & 0.320 \\
& Sig (bilateral) & $0.036^{*}$ \\
\hline
\end{tabular}

hs-CRP = high-sensitivity C-reactive protein; IL-6 = Interleukin-6; TNF- $\alpha=$ tumor necrosis factor alpha; ${ }^{*} p<0.05 ;{ }^{* *} p<0.01 ; * * * 00.001$. 
Table A.5

Correlation matrix between inflammatory biomarkers.

\begin{tabular}{llcccc}
\hline & & hs-CRP & IL-6 & TNF- $\alpha$ & Fibrinogen \\
\hline hs-CRP & Correlation coefficient & 1 & $.564^{* *}$ & .150 & $.589^{* *}$ \\
& Sig. (bilateral) & & .000 & .232 & .000 \\
IL-6 & Correlation coefficient & $.564^{* *}$ & 1 & .126 & $.488^{* *}$ \\
& Sig. (bilateral) & .000 & & .318 & .000 \\
TNF- $\alpha$ & Correlation coefficient & .150 & .126 & 1 & $.260^{*}$ \\
& Sig. (bilateral) & .232 & .318 & & .037 \\
\multirow{2}{*}{ Fibrinogen } & Correlation coefficient & $.589 * *$ & $.488^{* *}$ & $.260^{*}$ & 1 \\
& Sig. (bilateral) & .000 & .000 & .037 & \\
\hline
\end{tabular}

hs-CRP = high-sensitivity C-reactive protein; IL-6 = Interleukin-6; TNF- $\alpha=$ tumor necrosis factor alpha; $* 0<0.05 ;{ }^{* *} p<0.01$. 
Appendix A.6 - Backward multiple linear regression models exploring the influence of all inflammatory parameters with each significant cluster linked to an increase in BMI.

Table A.6.1

Backward regression models examining the link between the left LOC thickness and inflammation biomarkers.

\begin{tabular}{|c|c|c|c|c|c|c|c|}
\hline \multicolumn{2}{|c|}{ Model } & \multirow{2}{*}{$\frac{\beta}{0.19}$} & \multirow{2}{*}{$\frac{t}{1.23}$} & \multirow{2}{*}{$\frac{S E}{0.02}$} & \multirow{2}{*}{$\frac{p}{0.222}$} & \multirow[t]{2}{*}{${ }^{+} R^{2}$} & \multirow{2}{*}{$\begin{array}{l}\text { Sig. F } \\
\text { Change }\end{array}$} \\
\hline 1 & hs-CRP & & & & & & \\
\hline & IL-6 & -0.06 & -0.40 & 0.04 & 0.693 & \multirow{3}{*}{0.18} & \multirow{3}{*}{0.003} \\
\hline & TNF- $\alpha$ & -0.12 & -1.00 & 0.33 & 0.323 & & \\
\hline & Fibrinogen & -0.50 & -3.41 & 0.18 & $0.001 * *$ & & \\
\hline \multirow[t]{3}{*}{2} & hs-CRP & 0.16 & 1.18 & 0.02 & 0.242 & \multirow{3}{*}{0.19} & \multirow{3}{*}{0.693} \\
\hline & TNF- $\alpha$ & -0.12 & -1.00 & 0.32 & 0.319 & & \\
\hline & Fibrinogen & -0.52 & -3.62 & 0.17 & $0.001 * *$ & & \\
\hline \multirow[t]{2}{*}{3} & hs-CRP & 0.16 & 1.18 & 0.02 & 0.241 & \multirow{2}{*}{0.19} & \multirow{2}{*}{0.319} \\
\hline & Fibrinogen & -0.55 & -3.93 & 0.17 & $<0.001 * * *$ & & \\
\hline 4 & Fibrinogen & -0.45 & -3.99 & 0.14 & $<0.001 * * *$ & 0.19 & 0.241 \\
\hline
\end{tabular}

$L O C=$ lateral occipital cortex; $S E=$ standard error; ${ }^{+}$Adjusted $R$ squared value; $h s-C R P=$ highsensitivity C-reactive protein; IL-6 = Interleukin-6; TNF- $\alpha=$ tumor necrosis factor alpha; ${ }^{* *} p<$ $0.01 ; * * p<0.001$.

Table A.6.2

Backward regression models examining the link between the left fusiform thickness and inflammation biomarkers.

\begin{tabular}{|c|c|c|c|c|c|c|c|}
\hline \multicolumn{2}{|c|}{ Model } & \multirow{2}{*}{$\frac{\beta}{-0.10}$} & \multirow{2}{*}{$\frac{t}{-0.59}$} & \multirow{2}{*}{$\frac{S E}{0.02}$} & \multirow{2}{*}{$\frac{p}{0.559}$} & \multirow[t]{2}{*}{${ }^{+} R^{2}$} & \multirow{2}{*}{$\begin{array}{l}\text { Sig. F } \\
\text { Change }\end{array}$} \\
\hline 1 & hs-CRP & & & & & & \\
\hline & IL-6 & 0.06 & 0.42 & 0.05 & 0.675 & \multirow{3}{*}{0.06} & \multirow{3}{*}{0.115} \\
\hline & TNF- $\alpha$ & -0.02 & -0.18 & 0.35 & 0.855 & & \\
\hline & Fibrinogen & -0.30 & -1.89 & 0.19 & 0.064 & & \\
\hline \multirow[t]{3}{*}{2} & hs-CRP & -0.10 & -0.59 & 0.02 & 0.556 & \multirow{3}{*}{0.07} & \multirow{3}{*}{0.855} \\
\hline & IL-6 & 0.06 & 0.43 & 0.05 & 0.673 & & \\
\hline & Fibrinogen & -0.30 & -1.98 & 0.19 & 0.052 & & \\
\hline \multirow[t]{2}{*}{3} & hs-CRP & -0.07 & -0.47 & 0.02 & 0.644 & \multirow{2}{*}{0.08} & \multirow{2}{*}{0.673} \\
\hline & Fibrinogen & -0.29 & -1.95 & 0.18 & 0.056 & & \\
\hline 4 & Fibrinogen & -0.33 & -2.77 & 0.14 & $0.007 * *$ & 0.09 & 0.644 \\
\hline
\end{tabular}


Table A.6.3

Backward regression models examining the link between the right precentral thickness and inflammation biomarkers.

\begin{tabular}{|c|c|c|c|c|c|c|c|}
\hline \multicolumn{2}{|c|}{ Model } & \multirow{2}{*}{$\frac{\beta}{0.09}$} & \multirow{2}{*}{$\frac{t}{0.53}$} & \multirow{2}{*}{$\frac{S E}{0.02}$} & \multirow{2}{*}{$\frac{P}{0.600}$} & \multirow[t]{2}{*}{${ }^{+} R^{2}$} & \multirow[t]{2}{*}{$\begin{array}{c}\text { Sig. F } \\
\text { Change }\end{array}$} \\
\hline 1 & hs-CRP & & & & & & \\
\hline & IL-6 & -0.05 & -0.33 & 0.05 & 0.740 & \multirow{3}{*}{-0.002} & \multirow{3}{*}{0.428} \\
\hline & TNF- $\alpha$ & 0.01 & 0.08 & 0.36 & 0.935 & & \\
\hline & Fibrinogen & -0.27 & -1.64 & 0.20 & 0.106 & & \\
\hline \multirow[t]{3}{*}{2} & hs-CRP & 0.09 & 0.53 & 0.02 & 0.597 & \multirow{3}{*}{0.02} & \multirow{3}{*}{0.935} \\
\hline & IL-6 & -0.05 & -0.34 & 0.05 & 0.738 & & \\
\hline & Fibrinogen & -0.26 & -1.67 & 0.19 & 0.100 & & \\
\hline \multirow[t]{2}{*}{3} & hs-CRP & 0.07 & 0.44 & 0.02 & 0.664 & \multirow{2}{*}{0.03} & \multirow{2}{*}{0.738} \\
\hline & Fibrinogen & -0.28 & -1.82 & 0.19 & $0.074^{a}$ & & \\
\hline 4 & Fibrinogen & -0.24 & -1.94 & 0.15 & $0.057^{a}$ & 0.04 & 0.664 \\
\hline
\end{tabular}

Table A.6.4

Linear regression models examining the association between the left rostral middle frontal surface area and inflammation biomarkers.

\begin{tabular}{|c|c|c|c|c|c|c|c|}
\hline \multicolumn{2}{|c|}{ Model } & \multirow{2}{*}{$\frac{\beta}{0.27}$} & \multirow{2}{*}{$\frac{t}{1.65}$} & \multirow{2}{*}{$\frac{S E}{0.02}$} & \multirow{2}{*}{$\frac{p}{0.104}$} & \multirow[t]{2}{*}{${ }^{+} R^{2}$} & \multirow[t]{2}{*}{$\begin{array}{c}\text { Sig. F } \\
\text { Change }\end{array}$} \\
\hline 1 & hs-CRP & & & & & & \\
\hline & IL-6 & -0.13 & -0.87 & 0.05 & 0.387 & \multirow{3}{*}{0.04} & \multirow{3}{*}{0.175} \\
\hline & TNF- $\alpha$ & 0.04 & 0.28 & 0.35 & 0.780 & & \\
\hline & Fibrinogen & 0.13 & 0.83 & 0.19 & 0.412 & & \\
\hline \multirow[t]{3}{*}{2} & hs-CRP & 0.27 & 1.66 & 0.02 & 0.102 & \multirow{3}{*}{0.05} & \multirow{3}{*}{0.780} \\
\hline & IL-6 & -0.13 & -0.88 & 0.05 & 0.383 & & \\
\hline & Fibrinogen & 0.14 & 0.91 & 0.19 & 0.365 & & \\
\hline \multirow[t]{2}{*}{3} & hs-CRP & 0.22 & 1.44 & 0.02 & 0.156 & \multirow{2}{*}{0.06} & \multirow{2}{*}{0.383} \\
\hline & Fibrinogen & 0.12 & 0.73 & 0.18 & 0.469 & & \\
\hline 4 & hs-CRP & 0.28 & 2.31 & 0.01 & $0.024^{*}$ & 0.06 & 0.469 \\
\hline
\end{tabular}




\section{Table A.6.5}

Linear regression models examining the link between the right superior frontal surface area and inflammation biomarkers.

\begin{tabular}{|c|c|c|c|c|c|c|c|}
\hline \multicolumn{2}{|c|}{ Model } & \multirow{2}{*}{$\frac{\beta}{0.09}$} & \multirow{2}{*}{$\frac{t}{0.54}$} & \multirow{2}{*}{$\frac{S E}{0.02}$} & \multirow{2}{*}{$\frac{p}{0.593}$} & \multirow[t]{2}{*}{${ }^{+} R^{2}$} & \multirow[t]{2}{*}{$\begin{array}{c}\text { Sig. F } \\
\text { Change }\end{array}$} \\
\hline 1 & hs-CRP & & & & & & \\
\hline & IL-6 & -0.003 & -0.02 & 0.05 & 0.984 & \multirow{3}{*}{0.06} & \multirow{3}{*}{0.107} \\
\hline & TNF- $\alpha$ & 0.30 & 2.36 & 0.35 & $0.021 *$ & & \\
\hline & Fibrinogen & 0.05 & 0.30 & 0.19 & 0.765 & & \\
\hline \multirow[t]{3}{*}{2} & hs-CRP & 0.09 & 0.58 & 0.02 & 0.564 & \multirow{3}{*}{0.07} & \multirow{3}{*}{0.984} \\
\hline & TNF- $\alpha$ & 0.30 & 2.38 & 0.34 & $0.020^{*}$ & & \\
\hline & Fibrinogen & 0.05 & 0.31 & 0.18 & 0.761 & & \\
\hline \multirow[t]{2}{*}{3} & hs-CRP & 0.11 & 0.93 & 0.01 & 0.356 & \multirow{2}{*}{0.09} & \multirow{2}{*}{0.761} \\
\hline & TNF- $\alpha$ & 0.31 & 2.52 & 0.33 & $0.014^{*}$ & & \\
\hline 4 & TNF- $\alpha$ & 0.32 & 2.70 & 0.33 & $0.009 * *$ & 0.09 & 0.356 \\
\hline
\end{tabular}

